\title{
Fuzzy Multi-Objective Lattice Order Decision Approach for Preference Ranking in Conflict Analysis
}

\author{
Qiang Guo* and Wenyi Wang \\ School of Economics and Management, Southwest Jiaotong University, Chengdu 610031, China
}

Received 17 December 2013

Accepted 28 March 2016

\begin{abstract}
Based on conflict analysis and lattice order theory, this paper integrates fuzziness and non-transitivity of the preference, exclusiveness and incommensurability of the objective into conflict analysis, proposes a new fuzzy multi-objective lattice order decision method for preference ranking in conflict analysis. Furthermore, a conflict case study of Chinese enterprise overseas acquisition problem, including two players and complicated preference, is used to illustrate how the proposed method can be applied in practice along with its the feasibility and effectiveness. Conclusions showed that, compared to the traditional preference ranking in conflict analysis, the present method tends to be simpler, easier to reach an alliance and improve the stability of conflict situations. It also can avoid emotional and subjective decision-making, thus making results more rational and objective.
\end{abstract}

Keywords: conflict analysis; lattice order decision-making; preference ranking; the closeness of state.

\section{Introduction}

Conflict can be seen everywhere in our daily life, it comes from the opposition of objectives. Fraser and Hipel proposed conflict analysis theory based on metagame theory, which not only enriched the classical game theory, but also greatly improved the practicability of conflict study, thus attracted wide attention. The current research on conflict analysis, on the one hand, is focused on finding an alliance to improve stability of the situation and realizing equilibrium. On the other hand, some new methods are proposed based on the classical game theory, such as hyper-game ${ }^{[2]}$, the soft game ${ }^{[3]}$ and graph model ${ }^{[4]}$. The diversification and integrative conflict analysis approachs are also fruitful, such as rough set theory ${ }^{[5-6]}$, analytic hierarchy process $(\mathrm{AHP})^{[7]}$, matrix analysis ${ }^{[8-}$ ${ }^{10]}$, multi-objective game analysis ${ }^{[11]}$, Bayesian belief networks ${ }^{[12]}$, gray system theory ${ }^{[13]}$ and fuzzy analysis method ${ }^{[14-16]}$. Conflict analysis has been extensively investigated in the urban development planning, environmental resource disputes, project developing, as well as international military and trade disputes, etc.

In fact, the resolution of conflict is often subject to complex decision-making environment. For one thing, players often hesitate to choose the "best" or "the most satisfied", and even fallen into the dilemma of "you can't have your cake and eat it too". For another thing, due to the limited information and the limited ability of evaluation, players are often not clear about the value of things and tend to represent their cognition by linguistic language, which makes it difficult to get a clear and complete preference ranking. Players' preference ranking based on the imprecise and incomplete information has become an important research problem, it is necessary to consummate players' decision-making concept, and solve complex problems of "incommensurability" and "uncertain", to make their judgment more rational and objective.

${ }^{*}$ Corresponding author. 
Players are undoubtedly the most influential element conflict analysis, whose actions mainly depend on the preference. Researchers have different perspectives on players' preferences, including strength of preference $^{[17]}$, hybrid preference ${ }^{[18]}$, fuzzy preference and uncertain preference ${ }^{[19-23]}$, and players' emotions and attitudes ${ }^{[24-25]}$. Nevertheless, only a few findings have considered preference ranking, among which most methods still rely on the subjective objectives of players $^{[26]}$.

Preference is one of key factors that determine the development of the conflict. In reality, the preference perception of players is often subject to ambiguity and multi-objective problems. To solve this problem, researchers have made some further efforts, such as proposed mutiple preference ranking approachs from different angles ${ }^{[21]}$, or two-goal game model of conflict analysis based on game theory and multi-objective programming $^{[11]}$; or put forward two ranking methods with fuzzy preference information and the characteristic of multi-objective to overcome the bias of decision making ${ }^{[27-28]}$. But the perspectives of these methods seem to be limited, none of which get a fundamental solution to the problems of fuzziness and nontransitivity of the preference, as well as exclusiveness and incommensurability of the objective in conflict analysis.

Some people try to seek for new perspectives. To weaken the prerequisite that the player need to index all feasible state, a two-level drama model is built by adding a coordinator who represents general interests to get overall optimal resolution, but it fails to explain how to set the new coordinator ${ }^{[29]}$. A solution called the preference distribution based group decision-making is proposed, which uses the complete asymmetry preordering method to show the intensity of preference of players and get preferred alternatives set from possible alternatives set. The downside is that the method is based on a given preference distribution ${ }^{[30]}$. By combining grey system theory with conflict analysis, a grey conflict analysis model is bulit, which reflects players' satisfaction by the preference function and express gains and losses by grey interval numbers, but this evaluation is still based on players' experience, in reality, players are unlikely to be able to specify the interval value, thus the method seems to be less comprehensive and applicable ${ }^{[13]}$. Futhermore, hypergame analysis with the preference behavior is investigated, but it focuses on changes of preference utility in the dynamic evolution of the conflict and aims to analyze state transition rules to achieve stability. However, it's still based on the incommensurability and non-transitivity of preference ${ }^{[31]}$.

Accordingly, the characteristics of traditional preference ranking approaches in conflict analysis can be summarized as follows: 1) the preference ranking relys more on the players' standpoint, needs and wants, the likes and dislikes, using the "qualitative description" method; 2) they assume that the player is rational, with rich experience and knowledge, as well as strict thinking ability, who can make the right decisions in conflict analysis; 3) they also suppose that preference ranking meet the total order structure, where the pros and cons of the feasible state can be compared with each other. Actually, the above researches remains deficiencies in reflecting players' wishes, therefore the description of preferences seem to be impractical. The more common situation in conflict analysis is that players can only obtain the relative preference but seldom remain the complete rational, they even don't know where to start because of non-transitivity of the preference and contradictoriness of the objective.

In addition, preference ranking of conflict analysis has some characteristics with fewer schemes but higher risk. Actions are directly related to the evolution of conflict situations, hence it is necessary to optimize ranking methods. Lattice order is a kind of order structure between partial order and total order, which copes well with the non-transitivity in decision theory and make the incommensurability, unclear and uncertain preferences ordered and structured. The present work integrates fuzziness and non-transitivity of the preference, exclusiveness and incommensurability of the objective into the conflict analysis, and then proposes a new fuzzy multi-objective lattice order decision method for preference ranking in conflict analysis. Finally, its feasibility and effectivity are evaluated and illustrated in a real world case study.

The rest of the paper is organized as follows: Section 2 provides problem description. The new fuzzy multiobjective lattice order decision method for preference ranking in conflict analysis is proposed in Section 3. A conflict case study is provided in Section 4 to illustrate the proposed method in terms of feasibility and effectiveness. The paper is concluded in Section 5. 


\section{Problem Description}

Preference ranking of conflict analysis, in essence, is a multi-objective decision-making problem with a dynamic and multi-stage characteristic in reality. For the same objective, the players at different times have different preferences, and thus research on conflict analysis is often time related. With the situation changes, the model needs rebuilding.

Table 1. Definitions of variables in the model

\begin{tabular}{|c|c|}
\hline Variables & Definitions \\
\hline$N=\{1,2, \cdots, n\}$ & $\begin{array}{l}\text { The number of players in } \\
\text { conflict situations }\end{array}$ \\
\hline$O=\left\{o_{1}, o_{2}, \cdots, o_{i}\right\}$ & $\begin{array}{l}\text { The set of players' } \\
\text { objectives }\end{array}$ \\
\hline$T=\left\{t_{1}, t_{2}, \cdots, t_{k}\right\}$ & $\begin{array}{l}\text { The set of players' } \\
\text { feasible actions }\end{array}$ \\
\hline$S=\left\{s(T)_{1}, s(T)_{2}, \cdots, s(T)_{j}\right\}$ & $\begin{array}{l}\text { The set of players' } \\
\text { feasible states }\end{array}$ \\
\hline $\begin{array}{c}\tilde{W}=\left\{\tilde{W}_{1}, \tilde{W}_{2}, \cdots, \tilde{W}_{i}\right\} \text {, and } \\
\sum_{m=1}^{i} \tilde{W}_{m}=1\end{array}$ & Fuzzy objective weights \\
\hline$\tilde{X}=\left(\tilde{\mathrm{x}}_{i j}\right)_{i \times j}$ & $\begin{array}{l}\text { Fuzzy utility matrix of } \\
\text { feasible states }\end{array}$ \\
\hline$\tilde{U}=\tilde{X} \times \tilde{W}$ & $\begin{array}{l}\text { Fuzzy weighted utility } \\
\text { matrix of feasible states }\end{array}$ \\
\hline$\tilde{F}=\left\{U_{1}, U_{2}, \cdots, U_{j}\right\}$ & $\begin{array}{l}\text { Fuzzy multi-objective } \\
\text { preference matrix }\end{array}$ \\
\hline
\end{tabular}

In a conflict situation, players and their objectives are established conditions. Meanwhile, undetermined conditions are as follows: 1) feasible actions: the strategies that players may take to protect their interests; 2) feasible states: the results that consist of the allfeasible actions; 3) fuzzy objective weights: the fuzzy judgement of players towards different objectives, and 4) fuzzy utility values: the evaluation of players on each feasible state. To simplify the problem, the following variables and definitions are introduced in Table 1.

\section{Fuzzy Multi-Objective Lattice Order Decision Method for Preference Ranking in Conflict Analysis}

\subsection{Expression of fuzzy multi-objective preferences}

Players usually evaluate the satisfaction of feasible states by two ways: quantitative (e.g., numerical data) and qualitative (linguistic term). The former can be directly obtained from actual data, but the latter is a kind of subjective perception and needs to be transformed into fuzzy numbers. Due to the defects in triangular fuzzy numbers, which uses point values to express linguistic term with some errors, we choose the trapezoidal fuzzy numbers with good properties of approximate calculation to describe fuzzy evalution to make the results more practical.

Firstly, for all of the feasible states, the evaluation can be majorly divided into seven levels with specific transforming relations shown in Table 2, where there are three kinds of evaluations: revenue-based evaluation, cost-based evaluation and evaluation of weights.

Table 2. Transformation rules between linguistic evaluations and fuzzy numbers

\begin{tabular}{cccc}
\hline $\begin{array}{c}\text { Trapezoidal fuzzy } \\
\text { numbers }\end{array}$ & $\begin{array}{c}\text { Cost-oriented } \\
\text { evaluation }\end{array}$ & $\begin{array}{c}\text { Revenue- oriented } \\
\text { evaluation }\end{array}$ & $\begin{array}{c}\text { Evaluation of } \\
\text { weights }\end{array}$ \\
\hline$(0,0,0,0.2)$ & Very high (VH) & Very low (VL) & Absolutely unimportant (AU) \\
$(0,0,0.1,0.3)$ & High $(\mathrm{H})$ & Low (L) & Less important (U) \\
$(0,0.2,0.2,0.4)$ & Slightly high (SH) & Slightly low (SL) & Medium (M) \\
$(0.3,0.5,0.5,0.7)$ & Medium (M) & Medium (M) & Important (I) \\
$(0.6,0.8,0.8,1)$ & Slightly low (SL) & Slightly high (SH) & More important (MI) \\
$(0.7,0.9,1,1)$ & Low (L) & High (H) & Absolutely important (AI) \\
$(0.8,1,1,1)$ & Very low (VL) & Very high (VH)
\end{tabular}


According to the reference ${ }^{[32]}$, using the trapezoidal fuzzy numbers to represent the weights and the evaluations, then we can have

$$
\tilde{X}=\left(\tilde{\mathrm{x}}_{i j}\right)_{i \times j} \text { and } \tilde{W}=\left\{\tilde{W}_{1}, \tilde{W}_{2} \ldots \ldots \tilde{W}_{i}\right\} .
$$

Secondly, we use the following methods to normalize fuzzy utility matrix and fuzzy objective weights.

(1) The normalization of fuzzy utility matrix. Assume there is a trapezoidal fuzzy evaluation value:

$$
\tilde{X}_{i}^{\prime}=\left(\mathrm{a}_{i}, \mathrm{~b}_{i}, \mathrm{c}_{i}, \mathrm{~d}_{i}\right), i=1,2, \ldots m,
$$

where $m$ is the number of trapezoidal fuzzy evaluations. And let

$$
(\bullet)_{i}^{\max }=\max _{i}\left\{(\bullet)_{i}\right\} .
$$

Then the revenue-based evaluation can be normalized as:

$$
\tilde{X}_{\mathrm{i}}^{R}=\left(\frac{\mathrm{a}_{i}}{\mathrm{~d}_{\mathrm{i}}^{\max }}, \frac{\mathrm{b}_{i}}{\mathrm{c}_{\mathrm{i}}^{\max }}, \frac{\mathrm{c}_{i}}{\mathrm{~b}_{\mathrm{i}}^{\max }} \wedge 1, \frac{\mathrm{d}_{i}}{\mathrm{a}_{\mathrm{i}}^{\max }} \wedge 1\right) .
$$

Similarly, set

$$
(\bullet)_{i}^{\min }=\min _{i}\left\{(\bullet)_{i}\right\} \cdot
$$

The normalization of cost-based evaluation is then obtained by

$$
\tilde{X}_{\mathrm{i}}^{\mathrm{c}}=\left(\frac{\mathrm{a}^{\min } i}{\mathrm{~d}_{\mathrm{i}}}, \frac{\mathrm{b}^{\mathrm{min}}}{\mathrm{c}_{\mathrm{i}}}, \frac{c^{\min }{ }_{i}}{\mathrm{~b}_{\mathrm{i}}} \wedge 1, \frac{\mathrm{d}^{\mathrm{min}}}{\mathrm{a}_{\mathrm{i}}} \wedge 1\right) .
$$

(2) The normalization of fuzzy objective weights ${ }^{[33]}$. We first need to remove the fuzziness:

$$
W_{j}^{\prime}=\frac{\mathrm{c}_{j}+\mathrm{d}_{j}+\gamma_{j}+\delta_{j}}{4},
$$

which transforms fuzzy objective weights to the precise weights. Then according to

$$
W_{j}=\frac{W_{j}^{\prime}}{\sum_{m=1}^{i} W_{m}^{\prime}},
$$

we can obtain

$$
W=\left\{W_{1}, W_{2}, \cdots, W_{i}\right\} .
$$

Thirdly, the fuzzy utility value of each feasible state can be calculated by

$$
\tilde{U}_{i j}=\tilde{x}_{i j} \times W_{j} .
$$

Fuzzy multi-objective preference matrix is defined as

$$
\tilde{F}=\left\{U_{1}, U_{2}, \cdots, U_{j}\right\}
$$

\subsection{Lattice order decision algorithm for players' preference ranking}

According to the utility value of fuzzy multi-objective preference matrix for each feasible state, a lattice order decision-making method is proposed to get preference ranking. The main idea can be summed up in two cases: 1) for a finite lattice, we can directly select the top element as the optimal choice to determine order structure; 2) for the case of an unbounded lattice, we need to construct a virtual supremum and infimum, making which be the comparison standard and getting an ordering structure that meets comparative axioms. In the new ordering relation, the closeness of the feasible states can be measured so as to make reasonable decisions ${ }^{[34]}$. Specific algorithms are as follows:

Step 1: letting $R$ be the preference relation of players, and previous studies usually suppose $R \in\{\succ,<, \|\}, \quad$ here, the preference relation can be defined as $R \in\{>, \preccurlyeq, \sim, \|\}$. Among them, " $>$ " represents "superior to"; " $<$ " means "inferior to"; " $\sim$ " stands for "equal to"; while " $\|$ " expresses incommensurable binary relation, and lattice order decision is just aiming to solve these problems.

Step 2: Finding "the best"

$$
\tilde{U}^{+}=\left(\tilde{U}_{1}^{+}, \tilde{U}_{2}^{+}, \cdots, \tilde{U}_{j}^{+}\right)
$$

and "the worst"

$$
\tilde{U}^{-}=\left(\tilde{U}_{1}^{-}, \tilde{U}_{2}^{-}, \cdots, \tilde{U}_{j}^{-}\right)
$$

from the fuzzy multi-objective preference matrix

$$
\tilde{F}=\left\{U_{1}, U_{2}, \cdots, U_{j}\right\}
$$

under relations of the "maximum" and "minimum". Then we construct a lattice in the preference structure and draw the initial Hasse diagram, forming a preference structure to figure out whether the states are incommensurable.

Step 3: Using Eq. (1) and Eq. (2) to calculate the fuzzy utility value of all feasible states, namely, the 
distance between which and the best state, the worst state can be expressed as $D^{+}$and $D^{-}$. For the closeness of each feasible state, we use the Minkowski distance to measure that is simpler and more flexible, calculating the synthetic difference when $q=1$ and $q=2$. More specifically, the closeness is calculated by:

$$
\begin{aligned}
D_{j}^{+} & =\sqrt{\sum_{j}^{n}\left[d\left(\tilde{U}_{j}, \tilde{U}^{+}\right)\right]^{2}} \\
D_{j}^{-} & =\sqrt{\sum_{j}^{n}\left[d\left(\tilde{U}_{j}, \tilde{U}^{-}\right)\right]^{2}} \\
d\left(\tilde{U}^{+}, \tilde{U}^{-}\right) & =\left[\sum_{j=1}^{n}\left|\tilde{U}^{+}-\tilde{U}^{-}\right|^{q}\right]^{1 / q}
\end{aligned}
$$

Step 4: Calculating the synthetic closeness of each feasible state $D_{j}$ between the best and the worst, which is given as:

$$
\begin{aligned}
& D_{j}=p \frac{D_{j}^{-}}{D}+(1-p)\left(1-\frac{D_{j}^{+}}{D}\right) j=1,2, \cdots, n \\
& D=\sqrt{\sum_{j}^{n}\left[d\left(\tilde{U}^{+}, \tilde{U}^{-}\right)\right]^{2}}
\end{aligned}
$$

Among them, the bigger the value of $D_{j}$ is, the better the state performs. $D$ represents the distance between "the best" and "the worst", where $p$ is a kind of optimistic coefficient that indicates players' attitude towards feasible states, which does not affect the preference ranking and can be adjusted in accordance with the situation. Generally, $p$ value will only affect the closeness, the bigger it is, the greater closeness is, and therefore we usually take compromise to let $p=0.5$.

\section{A Case Study}

In this section, the proposed approach is applied into a practical problem - Chinese enterprise overseas acquisition conflict. The issue of enterprises overseas investment has been a major social concern. Under the background of economic globalization, foreign trade and economic cooperation undoubtedly provide convenience for the economic development of countries. Chinese enterprises have been committed to expanding the international market in recent years, but this road seems to be very hard and bumpy. In 2011, a telecom equipment manufacturer in China, trying to buy a small company abroad, but this small business ended in a failure because of a big transaction charge -"endangering national security", causing the world's major media attention and comments. We have to ponder the failure of acquisition, in addition to the government's political behavior factor; problems may largely lie in strategies from an enterprise perspective. Based on this incident, this present work takes Chinese

\begin{tabular}{|c|c|c|}
\hline Players & Options & Objectives \\
\hline \multirow{4}{*}{$\mathrm{MC}$} & $t_{1}:$ apply for the acquisition & $o_{1}:$ business opportunities and economic \\
\hline & $t_{2}:$ make efforts to achieve & benefits after the acquisition \\
\hline & the acquisition & $O_{2}: \cos t$ \\
\hline & $t_{3}:$ give up the acquisition & $O_{3}:$ corporate social image \\
\hline \multirow{3}{*}{ NG } & $t_{4}:$ agree to the acquisition & $\begin{array}{l}O_{4} \text { : business opportunities and economic } \\
\text { benefits after the acquisition }\end{array}$ \\
\hline & $\begin{array}{l}t_{5}: \text { agree to the acquisition } \\
\text { after consultation }\end{array}$ & $O_{5}:$ the image and credibility of the \\
\hline & $t_{6}:$ prevent the acquisition & $O_{6}:$ national security \\
\hline
\end{tabular}
enterprises overseas acquisition for example to verify the effectiveness of fuzzy multi-objective lattice order preference model in conflict analysis.

Table 3. Players' options and objectives 
Table 4. Feasible states for the acquisition conflict

\begin{tabular}{cccccccc}
\hline Players & Options & \multicolumn{7}{c}{ Feasible states } \\
\hline \multirow{3}{*}{ MC } & $t_{1}$ & 1 & 1 & 1 & 0 & 0 & 0 \\
& $t_{2}$ & 0 & 0 & 0 & 1 & 1 & 0 \\
& $t_{3}$ & 0 & 0 & 0 & 0 & 0 & 1 \\
& $t_{4}$ & 1 & 0 & 0 & 0 & 0 & 0 \\
NG & $t_{5}$ & 0 & 1 & 0 & 1 & 0 & 0 \\
& $t_{6}$ & 0 & 0 & 1 & 0 & 1 & 1 \\
& State number & $s_{1}$ & $s_{2}$ & $s_{3}$ & $s_{4}$ & $s_{5}$ & $s_{6}$ \\
\hline
\end{tabular}

\subsection{Modeling and solutions}

Five stakeholders are involved in the incident: the direct stakeholder of acquirer telecom equipment manufacturer $\mathrm{M}$ and acquiree company $\mathrm{N}$, the indirect stakeholder of Chinese government and the government in country $\mathrm{N}$, as well as the third-party participant of the investment committee in country $\mathrm{N}$. But the key decision-makers can be simplified in reality, and thus two players are considered in the model: telecom equipment manufacturer $\mathrm{M}$ company (MC), N government (NG). Players both have their own interests of objectives and strategies, which are summarized by the real data. Therefore, A conflict analysis model was developed with two players, and each player controlling three options and three objectives as shown in Table 3, where a brief explanation is furnished for each option and the objective controlled by the corresponding player.

For MC, in addition to the option of the application to acquire, they can take a series of efforts to avoid onevote veto of NG. For instance, paying attention to the attitude of NG, MC can take immediate remedial actions once they having a tendency of veto, specifically, strengthening communication with each policy makers and regulatory authorities in country $\mathrm{N}$ and persuading them actively, promoting and introducing the benefits of investment, as well as the promise that never steps into the field of national security information.

Table 5. Players' evaluation of feasible states and objective weights

\begin{tabular}{|c|c|c|c|c|c|c|c|c|}
\hline \multirow{2}{*}{\multicolumn{2}{|c|}{ Objectives }} & \multicolumn{6}{|c|}{ Feasible states } & \multirow{2}{*}{$\begin{array}{c}\text { Objective } \\
\text { weights }\end{array}$} \\
\hline & & $S_{1}$ & $s_{2}$ & $s_{3}$ & $S_{4}$ & $S_{5}$ & $S_{6}$ & \\
\hline \multirow{3}{*}{ MC } & $o_{1}$ & VH & $\mathrm{H}$ & $\mathrm{L}$ & $\mathrm{H}$ & $\mathrm{L}$ & $\mathrm{VH}$ & AI \\
\hline & \multirow{2}{*}{$\begin{array}{l}O_{2} \\
O_{3}\end{array}$} & $\mathrm{~L}$ & $\mathrm{SH}$ & M & $\mathrm{SH}$ & $\mathrm{H}$ & $\mathrm{L}$ & I \\
\hline & & $\mathrm{VH}$ & $\mathrm{H}$ & SL & VH & M & $\mathrm{L}$ & M \\
\hline \multirow{3}{*}{ NG } & $O_{4}$ & $\mathrm{H}$ & $\mathrm{H}$ & $\mathrm{L}$ & $\mathrm{H}$ & $\mathrm{L}$ & $\mathrm{L}$ & I \\
\hline & $O_{5}$ & $\mathrm{VH}$ & VH & $\mathrm{L}$ & VH & SL & M & M \\
\hline & $o_{6}$ & $\mathrm{~L}$ & $\mathrm{M}$ & $\mathrm{VH}$ & $\mathrm{H}$ & VH & $\mathrm{VH}$ & AI \\
\hline
\end{tabular}




$$
\begin{aligned}
& \tilde{X}_{M C}{ }^{\prime}=\left[\begin{array}{cccccc}
(0.8,1,1,1) & (0.7,0.9,1,1) & (0,0,0.1,0.3) & (0.7,0.9,1,1) & (0,0,0.1,0.3) & (0,0,0,0.2) \\
(0.6,0.8,0.8,1) & (0,0.2,0.2,0.4)(0.3,0.5,0.5,0.7)(0,0.2,0.2,0.4) & (0,0,0.1,0.3) & (0.7,0.9,1,1) \\
(0.8,1,1,1) & (0.7,0.9,1,1) & (0,0.2,0.2,0.4) & (0.8,1,1,1) & (0.3,0.5,0.5,0.7) & (0,0,0.1,0.3)
\end{array}\right]
\end{aligned}
$$

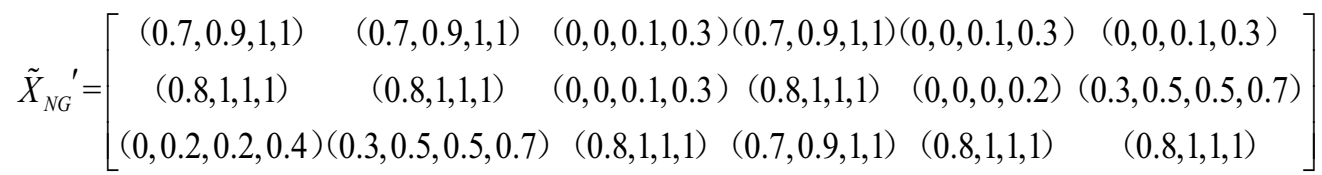

$$
\begin{aligned}
& \tilde{F}_{M C}=\left[\begin{array}{ccc}
(0.323,0.404,0.404,0.404) & (0.283,0.364,0.404,0.404) & (0,0,0.404,0.152) \\
(0,0,0.048,0.192) & (0,0,0.192,0.383) & (0,0,0.077,0.383) \\
(0.17,0.213,0.213,0.213) & (0.149,0.191,0.213,0.213) & (0,0.043,0.043,0.106)
\end{array}\right. \\
& \left.\begin{array}{ccc}
(0.283,0.364,0.404,0.404) & (0,0,0.404,0.152) & (0,0,0,0.101) \\
(0,0,0.192,0.383) & (0,0,0.383,0.383) & (0,0,0.043,0.164) \\
(0.17,0.213,0.213,0.213) & (0.064,0.106,0.106,0.186)(0,0,0.021,0.798)
\end{array}\right] \\
& \tilde{F}_{N G}=\left[\begin{array}{ccc}
(0.268,0.345,0.383,0.383) & (0.268,0.345,0.383,0.383) & (0,0,0.043,0.164) \\
(0.17,0.213,0.213,0.213) & (0.17,0.213,0.213,0.213) & (0,0,0.021,0.08) \\
(0,0.081,0.081,0.202) & (0.121,0.202,0.202,0.354)(0.323,0.404,0.404,0.404)
\end{array}\right. \\
& (0.268,0.345,0.383,0.383) \quad(0,0,0.043,0.164) \quad(0,0,0.043,0.164) \\
& (0.17,0.213,0.213,0.213) \quad(0,0,0,0.053) \quad(0.064,0.106,0.106,0.186) \\
& (0.283,0.364,0.404,0.404)(0.323,0.404,0.404,0.404)(0.323,0.404,0.404,0.404)
\end{aligned}
$$

Additionally, MC can increase public exposure and draw social support by means of the news media, so as to reduce suspicion and obstacles. These measures do have a certain effect on the attitude of NG, but which are directly related to final cost of inputs, and therefore it's necessary to consider objectives $\mathrm{O}_{2}$ when making decision.

$\mathrm{MC}$ is one of the important enterprises in China, so the conflict is essentially an interest game between the two countries in the high-tech industry rather than the enterprise trade dispute. For NG, they are always sensitive and cautious about every investment behavior that may threaten national security, and attach great importance to that. However, NG has ever promised to treat the trade cooperation with Chinese enterprises fairly in the bilateral strategic dialogue, and advocating free and fair competition, thus the Objective $O_{5}$ of national image and government credibility also need to be taken into account. Considering various factors, NGs may choose $t_{5}$ after the scrutiny of the acquisition and efforts of MC making for the acquisition. As long as the acquisition does not involve national security information, the cooperation can be achieved.

Depending on feasible actions, the set of feasible states will be generated after players' analyzing. The results are given in Table 4, where a "1" indicates that the action is selected by the controlling player and a" 0 "stands for the corresponding action is not chosen by the player. In accordance with the conflict situation, the performance that all feasible objectives match the objectives and the evaluation of objective weights are given in Table 5.

First, we obtain the state and the objective fuzzy evaluation matrix of MC and NG by trapezoidal fuzzy numbers in Table 5:

$$
\tilde{X}_{\mathrm{MC}}^{\prime}, \tilde{X}_{\mathrm{NG}}^{\prime}
$$

As show above and the corresponding fuzzy weights matrix

$$
W_{M C}{ }^{\prime}, W_{N G}{ }^{\prime}
$$




$$
\tilde{W}_{M C}{ }^{\prime}=\left[\begin{array}{c}
(0.8,1,1,1) \\
(0.7,0.9,1,1) \\
(0.3,0.5,0.5,0.7)
\end{array}\right], \tilde{W}_{N G}^{\prime}=\left[\begin{array}{c}
(0.7,0.9,1,1) \\
(0.3,0.5,0.5,0.7) \\
(0.8,1,1,1)
\end{array}\right] .
$$

Secondly, using the method given in 3.1 to normalize $\tilde{X}_{\mathrm{MC}}{ }^{\prime}, \tilde{X}_{\mathrm{NG}}{ }^{\prime}$ and $W_{M C}{ }^{\prime}, W_{N G}{ }^{\prime}$ respectively, calculating the fuzzy weighted utility matrix by the equation:

$$
\tilde{U}=\tilde{X} \times \tilde{W} .
$$

Then we can obtain fuzzy multi-objective preference matrixes:

$$
\tilde{F}_{M C}, \tilde{F}_{N G} .
$$

At the same time, "the best" and "the worst" are determined by the method proposed in 3.2, which can be expressed as

$$
\tilde{U}_{M C}{ }^{+}, \tilde{U}_{M C}{ }^{-}, \tilde{U}_{N G}{ }^{+} \text {, and } \tilde{U}_{N G}{ }^{-},
$$

which are shown as below:

$$
\begin{gathered}
\tilde{U}_{M C}{ }^{+}=\left[\begin{array}{c}
(0.334,0.404,0.404,0.404) \\
(0,0,0.383,0.383) \\
(0.17,0.213,0.213,0.213)
\end{array}\right], \\
\tilde{U}_{M C}{ }^{-}=\left[\begin{array}{c}
(0,0,0,0.1) \\
(0,0,0.043,0.16) \\
(0,0,0.021,0.08)
\end{array}\right], \\
\tilde{U}_{N G}{ }^{+}=\left[\begin{array}{c}
(0.268,0.345,0.383,0.383) \\
(0.17,0.213,0.213,0.213) \\
(0.323,0.404,0.404,0.404)
\end{array}\right], \\
\tilde{U}_{N G}{ }^{-}=\left[\begin{array}{c}
(0,0,0.043,0.164) \\
(0,0,0,0.053) \\
(0,0.081,0.081,0.202)
\end{array}\right] .
\end{gathered}
$$

From the fuzzy multi-objective preference matrix $\tilde{F}_{M C}$ and $\tilde{F}_{N G}$, it can be seen that there exists two incommensurable conditions in the feasible states of $\mathrm{M}$ :

$$
S_{1}\left\|S_{2}\right\| S_{4} \text {, and } S_{3} \| S_{5} \text {. }
$$

Similarly, $\mathrm{N}$ has also encountered this problem in preference ranking:

$$
S_{1}\left\|S_{2}\right\| S_{4} \text {, and } S_{3}\left\|S_{5}\right\| S_{6} \text {. }
$$

The values that the feasible states match the objectives have a virtual supremum and infimum, thus we can get a Hasse diagram (Figure 1).

Using equation (1) and equation (2) to calculate the distance between feasible states and the best state, the worst states when $q=1$ and $q=2$, which can be defined as $D_{M C}{ }^{+}, D_{M C}{ }^{-}$and ${D_{N G}}^{+}, D_{N G}{ }^{-}$. Meanwhile, the synthetic closeness of the feasible state can be calculated by equation (3):

$$
D_{M C}, D_{N G} \text {, }
$$

the results are shown in Table 6 .
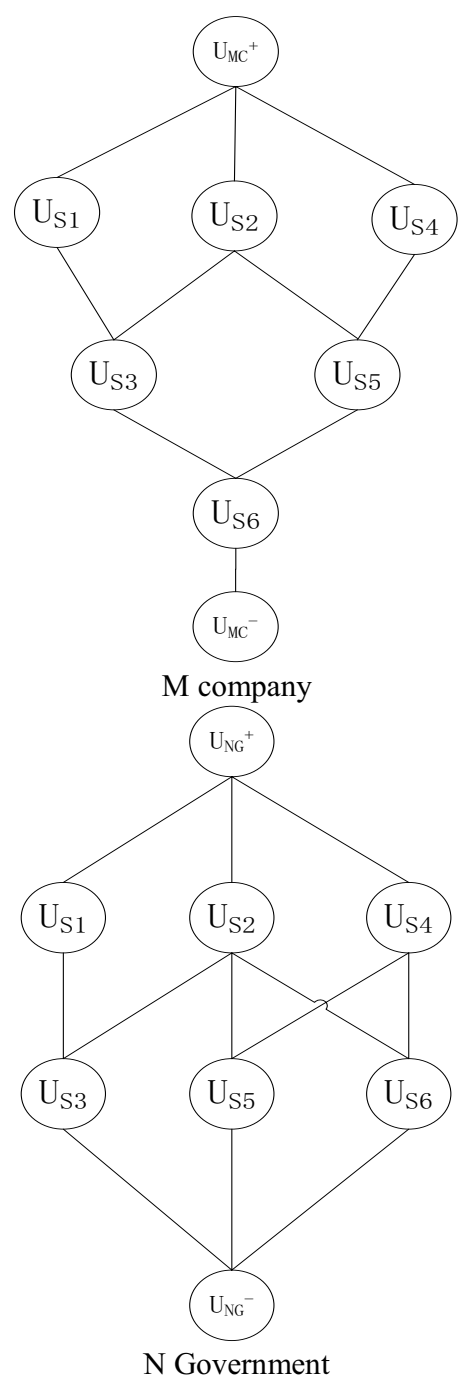

Fig. 1 Preliminary Hasse diagram of feasible state ranking 
From Table 6, there exists a same result of preference ranking when $q=1$ and $q=2$, and the synthetic closeness differs little between these two conditions. The bigger the synthetic closeness is, the better the state performs. Hence, the overall ranking of $\mathrm{MC}$ and $\mathrm{NG}$ are as follow respectively:

$$
\begin{aligned}
& s_{4} \succ s_{2} \succ s_{1} \succ s_{5} \succ s_{3} \succ s_{6} ; \\
& s_{4} \succ s_{2} \succ s_{1} \succ s_{6} \succ s_{3} \succ s_{5} ;
\end{aligned}
$$

\begin{tabular}{|c|c|c|c|c|c|c|c|}
\hline \multicolumn{2}{|c|}{ MC } & $D_{s 1}$ & $\mathbf{D}_{\mathrm{S} 2}$ & $\mathbf{D}_{\mathrm{S} 3}$ & $\mathbf{D}_{\mathrm{S} 4}$ & $\mathrm{D}_{\mathrm{S} 5}$ & $D_{\text {S6 }}$ \\
\hline \multirow{2}{*}{$D_{M C}^{+}$} & $\mathrm{q}=1$ & 0.527 & 0.315 & 2.268 & 0.272 & 1.69 & 2.702 \\
\hline & $q=2$ & 0.386 & 0.202 & 0.81 & 0.2 & 0.706 & 0.903 \\
\hline \multirow{2}{*}{$D_{M C}^{-}$} & $q=1$ & 2.175 & 2.387 & 0.434 & 2.429 & 1.012 & 0 \\
\hline & $q=2$ & 0.809 & 0.807 & 0.237 & 0.816 & 0.449 & 0 \\
\hline \multirow{2}{*}{$D_{M C}$} & $q=1$ & 0.801 & 0.877 & 0.175 & 0.892 & 0.383 & 0.01 \\
\hline & $q=2$ & 0.73 & 0.833 & 0.199 & 0.839 & 0.368 & 0.005 \\
\hline \multicolumn{2}{|c|}{ NG } & $D_{\text {s1 }}$ & $\mathbf{D}_{\mathrm{S} 2}$ & $\mathbf{D}_{\mathrm{S} 3}$ & $\mathrm{D}_{\mathrm{S} 4}$ & $\mathbf{D}_{\mathrm{S} 5}$ & $D_{\text {S6 }}$ \\
\hline \multirow{2}{*}{$D_{N G}^{+}$} & $q=1$ & 1.172 & 0.657 & 1.879 & 0.081 & 1.927 & 1.518 \\
\hline & $q=2$ & 0.596 & 0.354 & 0.695 & 0.057 & 0.707 & 0.624 \\
\hline \multirow{2}{*}{$D_{N G}^{-}$} & $q=1$ & 1.927 & 2.443 & 1.22 & 3.019 & 1.172 & 1.582 \\
\hline & $q=2$ & 0.707 & 0.753 & 0.596 & 0.897 & 0.596 & 0.632 \\
\hline \multirow{2}{*}{$D_{N G}$} & $q=1$ & 0.622 & 0.788 & 0.394 & 0.974 & 0.378 & 0.51 \\
\hline & $q=2$ & 0.56 & 0.716 & 0.447 & 0.955 & 0.44 & 0.504 \\
\hline
\end{tabular}

Table 6. Synthetic nearness, distance between feasible states and "the best" and "the worst"

\subsection{Result analysis and discussions}

According to the findings, the optimal choice is not $s_{1}$, but the state $s_{4}$ for MC, which is better prepared, more comprehensive consideration and more stable in contrast to others. As we all know, any acquisition of the enterprise cannot be plain sailing. So before applying for the acquisition, $\mathrm{MC}$ should make pre-preparation for the refusal of $\mathrm{NG}$, being aware of any possible resistance and take effective measures to resolve these problems as quick as possible. Therefore $s_{4}$ is undoubtedly more rational choice and having more advantages.

From NG's perspective, preventing this acquisition can protect the domestic economy and reduce threats to national security. However, under the current background of economic globalization, which is not really a wise choice and not only affects the government's reputation and image, but also hinders the long-term economy development. Therefore, it is sensible to make a compromise in both sides as much as possible. For instance, NG can ask for revising the acquisition and adding new provisions to protect national security information.

Comparing the preference ranking of both sides by traditional methods in reality:

$$
\begin{aligned}
& s_{1} \succ s_{4} \succ s_{2} \succ s_{3} \succ s_{6} \succ s_{5} ; \\
& s_{6} \succ s_{3} \succ s_{5} \succ s_{4} \succ s_{2} \succ s_{1} .
\end{aligned}
$$

Among which, MC believes it is desirable to apply for the acquisition directly without any remedial measures, while it is the worst choice for NG. It is difficult to achieve a balanced state and the cooperation. We can say, both sides have not taken a thoughtful action in the conflict, and they are dissatisfied with the final state essentially.

Therefore, it is not hard to see, using preference ranking obtained by the lattice order decision method is easier to bring the two sides to reach a settlement. When $\mathrm{MC}$ and NG have adopted preference ranking given in 
the present method, the conflict can turn to be an alliance. Reasons may lie in that traditional preference ranking of conflict analysis depends on players' subjective evaluation, and is likely to be irrational, vulnerable to some factors such as emotions and attitudes. The method proposed in this paper can exactly avoid this problem, not only coordinate multiple exclusive objectives, but also characterize players' subjective perception as objective as possible.

\section{Conclusions}

Finding an effective way to analyze and resolve conflict problems has been an important topic in our society. Currently, research on conflict analysis tends to be active in the academic field, and its methods gradually are applied to practical problems. However, most studies mainly focused on the realization of alliances and stability, paying more attention to results but the process - the importance of preference ranking to the evolution of conflict is neglected. A number of conflicts often become more sharpened because of errors in decision making, resulting in many unnecessary losses.

Actually, the result from this paper is a supplement to the research on preference ranking of conflict analysis, focusing on behavioral characteristics and complex cognitive of the players, using the lattice order decision method to settle these problems. On the one hand, this paper provided a new idea and method for the research and application in conflict analysis. On the other hand, some ideas are proposed to overcome limited rationality and reach an alliance for both sides. A case study of Chinese enterprises overseas acquisition conflict, including two major players, is used to illustrate how the proposed method can be applied in practice and its feasibility and effectiveness has been verified satisfiable, thereby providing strategic guidance for enterprises overseas trade friction and even conflict in other areas. On the surface, the preference ranking given in this paper may not be in the interests of the players, but in essence it is a thoughtful decisionmaking.

Further efforts can be made in the following aspects: 1) considering the situation there are multiple decision makers in each party, namely, introducing group decision in conflict analysis so as to make research be more practical; 2) considering the dynamic evolution of players' preference. Conflict is a dynamic multi-stage problem and the method given in this paper only aims at a certain point; 3) considering expected probability of feasible states, introducing probability and risk to better analyze players' behaviors; 4) computerized decisionmaking process. To facilitate an analyst to apply the proposed method, it would be worthwhile to investigate how to implement it into a decision support system and incorporate computer decision support system into lattice order decision for preference ranking.

\section{Acknowledgements}

The authors are very thankful for the reviewer's thoughtful suggestions to improve the paper. This research was financially supported by the National Natural Science Foundation of China (Grant No. 71340009).

\section{References}

1. D.M. Kilgour, K.W. Hipel, Negotiation support using the graph model for conflict resolution, Group Decision and Negotiation 3(1994) 29-46.

2. P.G. Bennett, Hypergames: developing a model of conflict, Futures 12(1980) 489-507.

3. J. Bryant, Drama theory: dispelling the myths, Journal of the Operational Research Society 58(5)(2007) 602613.

4. K.W. Hipel, D.M. Kilgour, L. Fang, X.Y. Peng, The decision support system GMCR in environmental conflict management, Applied Mathematics and Computation 83(1997) 117-152.

5. Z. Pawlak, Some remarks on conflict analysis, European Journal of Operational Research 166(2005) $649-654$.

6. J.S. Gao, D.Y. Gu, Z.Y. Xu, Y.W. Wu, J. Wei, New conflict analysis model based on rough set theory, Chinese Journal of Management Science 16(2)(2008) 150-156.

7. G.Y. Kea, K.W. Li, K.W. Hipel, An integrated multiple criteria preference ranking approach to the Canadian west coast port congestion conflict, Expert Systems with Applications 39(2012) 9181-9190.

8. H.Y. Xu, K.W. Hipel, D.M. Kilgour, Matrix representation of solution concepts in multiple decision maker graph models, IEEE Trans. Syst. Man Cybern.-Part A 39(1)(2009) 96-108.

9. H.Y. Xu, D.M. Kilgour, K.W. Hipel, G. Kemkes, Using matrices to link conflict evolution and resolution in a graph model, European Journal of Operational Research 207(2010) 318-329. 
10. H.Y. Xu, K.W. Li, K.W. Hipel, D.M. Kilgour, A matrix approach to status quo analysis in the graph model for conflict resolution, Applied Mathematics and Computation 212(2009) 470-480.

11. C.S. Lee, Multi-objective game-theory models for conflict analysis in reservoir watershed management, Chemosphere 87(6)(2012) 608-613.

12. R. Giordano, D. D'Agostino, C. Apollonio , N. Lamaddalena, M. Vurro, Bayesian belief network to support conflict analysis for groundwater protection: the case of the Apulia region, Journal of Environmental Management 115(2013) 136-146.

13. C. Li, Grey conflict analysis model based on uncertainty for enterprise strategy orientation. In Proceedings of the 16th National Grey System Conference, 2008, pp. 145-155.

14. G. Munda, A conflict analysis approach for illuminating distributional issues in sustainability policy, European Journal of Operational Research 194(2009) 307-322.

15. M.S. Almutairi, K.W. Hipel, M.S. Kamel, Fuzzy preference in conflicts, Journal of Systems Science and Systems Engineering 17(3)(2008) 257-276.

16. D.Y. Gu, J.S. Gao, Z.Y. Xu, F. Li, Conflict analyses model based on vague sets theory, Journal of Huazhong University of Science and Technology (Natural Science Edition - in Chinese) 36(3)(2008) 95-98.

17. L. Hamouda, D.M. Kilgour, K.W. Hipel, Strength of preference in graph models for multiple-decisionmaker conflicts, Applied Mathematics and Computation 179(2006) 314-327.

18. H.Y. Xu, D.M. Kilgour, K.W. Hipel, E.A. McBean, Theory and application of conflict resolution with hybrid preference in colored graphs, Applied Mathematical Modelling 37(2013) 989-100.

19. K.W. Li, T. Inohara, H.Y. Xu, Coalition analysis with preference uncertainty in group decision Support, Applied Mathematics and Computation 231(2014) 307-319.

20. H.Y. Xu, D.M. Kilgour, K.W. Hipel, Matrix representation and extension of coalition analysis in group decision support, Computers and Mathematics with Applications 60(2000) 1164-1176.

21. K.W. Li, K.W. Hipel, D.M. Kilgour, L. Fang, Preference uncertainty in the graph model for conflict resolution, IEEE Trans. Syst. Man Cybern. -Part A: Syst. Humans 34(4)(2004) 507-520.
22. K.W. Li, K.W. Hipel, D.M. Kilgour, D. Noakes, Integrating uncertain preference into status quo analysis with applications to an environmental conflict, Group Decision Negotiation 14(6)(2005) 461-479.

23. M.S. Almutairi, K.W. Hipel, M.S. Kamel, Fuzzy preference in conflicts, Journal of Systems Science and Systems Engineering, 17(3)(2008) 257-276.

24. S.B. Walker, K.W. Hipel, T. Inohara, Strategic decision making for improved environmental security: Coalitions and attitudes, Journal of Systems Science and Systems Engineering 18(4)(2009) 461-476.

25. A. Obeidi, K.W. Hipel, D.M. Kilgour, The role of emotions in envisioning outcomes in conflict analysis, Group Decision and Negotiation 14(6)(2005) 481500.

26. S.B. Su and Z.B. Hu, Conflict analysis between information sharing and webpage propriety in webpage of EC: evident from China, Journal of Software 5(11)(2010) 1300-1305.

27. B. Meng, A procedure of conflict and analysis with fuzzy preference information and its application, Systems Engineering 9(6)(1991) 36-41.

28. B. Meng, T. Chen, H. Fu, A procedure of conflict analysis with multi-objectives, Control and Decision 7(3)(1992) 182-193.

29. J.F. Cai, S.Y. Zhang, J.W. Xue, A two-level drama and its applications in conflict analysis, Systems Engineering 22(4)(2004) 95-99.

30. R.Z. Cao and Y.J. Li, A method of group decision making based on preference distribution, Journal of Management Engineering 18(3)(2004) 121-123

31. H.J. Yuan, Y.X. Song, Y. Qu, Hypergame analysis with players behavior preferences, Computer and Digital Engineering 41(3)(2013) 410-413.

32. R.J. Li, Fuzzy Multiple Attribute Decision Theory and Application. Beijing Scientific Press (in Chinese), 2002, pp. 138-198.

33. S.M. Chen, Evaluating weapon systems using fuzzy arithmetic operations, Fuzzy Sets and Systems 77(3) (1996) 265-276.

34. G. Hao, Q.F. Mou, D.Y. Zhang, Y.H. Guo, Approach of fuzzy multi-objective decision-making based on lattice-order preference. Journal of Southwest Jiaotong University (in Chinese) 41(4) (2006) 517521. 